\title{
TTR
}

Traduction, terminologie, rédaction

\section{Dénis de traduction et désir de traduire}

\section{Yves Gambier}

Volume 26, numéro 2, 2e semestre 2013

Traduction et conscience sociale. Autour de la pensée de Daniel Simeoni

Translation as Social Conscience. Around the Work of Daniel Simeoni

URI : https://id.erudit.org/iderudit/1037138ar

DOI : https://doi.org/10.7202/1037138ar

Aller au sommaire du numéro

\section{Éditeur(s)}

Association canadienne de traductologie

ISSN

0835-8443 (imprimé)

1708-2188 (numérique)

Découvrir la revue

Citer cet article

Gambier, Y. (2013). Dénis de traduction et désir de traduire. TTR, 26(2),

219-243. https://doi.org/10.7202/1037138ar

\section{Résumé de l'article}

La traduction connaît des transformations sous l'influence des TIC. Naguère, elle était invisible, niée, à la fois comme besoin, comme travail, comme profession et comme discipline. En quelques décennies, un nouvel environnement a bousculé ce monde de la traduction, laissant apparaître de nouveaux types de traducteurs et modifiant offres et demandes. Il n'empêche que nous avons encore besoin d'outils et de méthodes appropriés pour analyser les nouvelles hiérarchies entre d'une part les traducteurs et d'autre part les divers marchés du travail. 


\title{
Dénis de traduction et désir de traduire
}

\section{Yves Gambier}

Université de Turku

\section{Résumé}

La traduction connaît des transformations sous l'influence des TIC. Naguère, elle était invisible, niée, à la fois comme besoin, comme travail, comme profession et comme discipline. En quelques décennies, un nouvel environnement a bousculé ce monde de la traduction, laissant apparaître de nouveaux types de traducteurs et modifiant offres et demandes. Il n’empêche que nous avons encore besoin d'outils et de méthodes appropriés pour analyser les nouvelles hiérarchies entre d'une part les traducteurs et d'autre part les divers marchés du travail.

Mots-clés: dénis de traduction, tournant économique en traductologie, traducteurs amateurs, traduction et journalisme, traduction participative

\begin{abstract}
Translating and translation are transformed with Information and Communication Technology (ICT). Yesterday, translation was invisible, denied-as a need, as a process, as a profession, and as a discipline. Within three decades, a new work environment shakes up the translators' world. New types of translators emerge. The balance between supply and demand is changing. However, we still need adequate tools and methods to investigate the new hierarchy which has become established between translators, between different kinds of job markets.
\end{abstract}

Keywords: denials in translation, economic turn in translation studies, volunteer translators, translation and journalism, collective translation

\section{Introduction}

Y aurait-il un désir de traduire inné, compulsif qui ferait quon ne pourrait s'empêcher de passer à l'acte? Berman (1990, p. 6) a parlé de "pulsion de traduire», sans jamais vraiment creuser le concept. Il y a bien eu aussi quelques tentatives pour rapprocher traduction, traductologie et psychanalyse: le désir serait-il du domaine réservé de l'introspection psychique? Certes l'acte de traduire a une dimension psycho-somatique - non dans le sens 
de troubles organiques ou fonctionnels, mais dans la mesure où il est incorporé (avec stress, tension, rythme) et incorporant (le document à traduire est «assimilé », parfois jusqu'au cannibalisme). Notre problématique cependant, dans ce qui suit, ne relève pas de cette perspective. Elle est plutôt d'orientation sociologique, pour tenter d'appréhender comment et jusqu'où les diverses possibilités d'automatisation de la traduction qui satisfont déjà certains besoins plus ou moins volumineux et plus ou moins urgents ( $c f$. section 2) viennent remettre en cause la place et le rôle même de la traduction, et, surtout, comment elles transforment la perception qu'on en a.

Les apports des technologies de l'information et de la communication (TIC) modifient et vont certainement modifier nos attitudes envers la traduction ainsi que certaines de nos représentations. C'est la thèse principale de ce qui suit: ces modifications pourraient induire une rupture importante à la fois dans les pratiques et dans les discours tenus sur la traduction.

Afin de mieux mettre en perspective les changements, il faut rappeler que la traduction et l'interprétation ne sont qu'une des solutions possibles pour régler les relations et communications internationales multilingues. En effet, selon les époques et les rapports de pouvoir, d'autres moyens existent et sont valorisés différemment, comme l'apprentissage des langues, le bilinguisme passif, le recours à une lingua franca (Lambert, 1989, p. 233), mais il y a aussi des stratégies d'exclusion, comme la construction de murs et l'ethnocide. Ce bref rappel permet de replacer la traduction dans les enjeux des politiques linguistiques ${ }^{1}$ et de balayer toute naïveté quant à la croissance irrémédiable des demandes en traduction.

Notre argumentation s'établit en deux temps: le déni de la traduction et des traducteurs a perduré sous diverses formes pendant des siècles, mais il est bousculé, depuis près de trois décennies, par le nouvel environnement de travail. Dès lors, de nouveaux types de traducteurs apparaissent. Quelles seraient les conséquences à en titrer - sur l'appréhension de la traduction, sur le statut des traducteurs?

1. Les marchés de la traduction (littéraire, scientifique) sont au moins doublement structurés - par les aires linguistiques et par les États-nations -, les deux ne se recoupant pas nécessairement et étant eux-mêmes structurés entre centre et périphérie (par ex. au sein de la francophonie). 


\section{Les dénis de traduction}

La traduction, prise dans son sens traditionnel surtout appliqué aux textes sacrés et littéraires, a été niée, à la fois comme besoin, comme travail, comme profession et comme discipline. Toutes les langues et sociétés ne sont pas affectées de la même façon, au même degré, au même moment. Les remarques et réflexions cidessous sont donc à prendre avec prudence et à ne pas généraliser abusivement. Mais une historicisation détaillée des pratiques et des discours dépasse le cadre de cet article.

\subsection{Déni de la traduction comme besoin}

Pendant longtemps, la traduction a semblé ne servir que les pouvoirs en place, que les autorités établies (royales et religieuses), comme étant inexistante, occultée dans les échanges commerciaux, scientifiques, philosophiques (Delisle et Woodsworth, 2007). La non-traduction ou traduction occulte a été débattue (par exemple, par Toury, 1995, p. 23-39). Cette non-reconnaissance du statut de traduction pour certains documents, comme nombre de publicités et d'informations télévisées, rend difficile l'appréhension du volume de traductions réalisées. Il devient en effet plus ardu de connaître l'évolution des marchés ( $c f .4 .1$ ), avec leur logique, leurs instances et leurs agents, si on ne peut obtenir de données fiables sur les demandes (actuelles, prévisibles, latentes ou potentielles) et offres d'aujourd'hui (Gouadec, 2002, p. 87-115 ; Gouadec, 2007, p. 120-145). La traduction n'est-elle d'ailleurs qu'un besoin ou estelle une nécessité dans un monde globalisé? Entre les exigences de «survie» dans le flux des communications internationales et la satisfaction non essentielle qu'est la localisation d'une campagne publicitaire, les besoins en traduction peuvent-ils être hiérarchisés? Une telle hiérarchisation recouperait et justifierait le continuum entre professionnels et amateurs ( $c f .2 .2)$.

La non-reconnaissance de nombre de traductions prend un tour nouveau aujourd'hui avec la prolifération des étiquettes qui tendent à nier ou déprécier la «traduction» ou à éviter le recours au terme. Il ne s'agit pas ici et maintenant de nous arrêter sur les fondements de cet éclatement des dénominations (dans toutes les sociétés? dans toutes les langues?) touchant le transfert, le passage, le transculturel, tout un travail qui se paie, avec de l'argent, par de l'anonymat et de l'indifférence souvent. Je ne pense pas aux catégories traditionnelles comme la traduction spécialisée 
ou littéraire, l'interprétation de conférence ou de communauté, catégories qui relèvent de la tribu des traducteurs. Je pense aux étiquettes qui s'imposent dans divers milieux professionnels commanditaires, demandeurs de traduction -, mais qui nient le mot au profit, notamment, de : localisation, adaptation, documentation multilingue, editing, transediting, rédaction technique multilingue, médiation langagière, versionisation, révision, corédaction (de textes juridiques, par exemple), transcréation (Ray et Kelly, 2010), etc. La multiplication des fonctions à remplir en même temps (de documentation, de terminologie, de gestion de projet, de conception de sites, de relecture), la spécialisation poussée (par domaine, par outil, par type de document) «définissent des ensembles de savoirfaire et de compétences particulières en métiers de l'ingénieur en communication multilingue multimédia» (Gouadec, 2002, p. 70). Cet IC2M pourrait être la figure future du traducteur.

\subsection{Déni de la traduction comme activité à part entière}

Le déni a porté et porte encore sur la traduction comme travail et explique en partie la prolifération relative des dénominations. Nombre de commanditaires, d'amateurs, d'auto-traducteurs (universitaires), d'ingénieurs en industries de la langue continuent de penser que la traduction est un processus mécanique, le remplacement d'un mot par un autre, un problème de dictionnaires, qu'ils pourraient eux-mêmes effectuer s'ils en avaient le temps, mais qu'ils préfèrent souvent passer, non sans condescendance, à une cousine qui connaît les langues, à une secrétaire bilingue ou au pire à un traducteur professionnel, à condition qu'il n'exige pas trop financièrement. Une telle perception du texte à traduire comme suite linéaire de mots ou de phrases (conception qui recoupe en partie les offres et contraintes des logiciels de mémoire de traduction, $c f$. 2.2) explique sans doute qu'on ait longtemps considéré le statut de la traduction comme inférieur, subordonné, second par rapport à celui de l'original. ${ }^{2}$

Cela dit, combien l'idée de «traduction» et l'univers des traducteurs demeurent parfois archaïques aux yeux de beaucoup

2. Bien des outils d'aide à la traduction dérivent encore de la conception prédominante lors des premiers essais de traduction automatique (substitution de mot à mot). Mais cette propagation est peu à peu remplacée par de nouvelles approches, basées sur le contexte, la fréquence d'emploi, la remise en cause de la bi-univocité du signe linguistique ainsi que la convivialité (user-friendly) et l'utilisabilité de ces outils. 
qui vivent sur des archétypes, préférant concevoir la langue comme un objet statique plutôt que comme une dynamique, préférant envisager la communication comme un ensemble d'informations plutôt que comme une interaction, préférant affirmer qu'un message, c'est une concaténation de données plutôt que l'expression d'une identité. D'où les diatribes récurrentes sur "lost in translation», comme si les traducteurs ne pouvaient que niveler, neutraliser, affadir dans leur effort désespéré d'équivalence dans ce qui ne serait qu'une affaire de mots, sans enjeux, sans complexité culturelle. D'où aussi l'exhibition fréquente d'exemples de «mauvaises» traductions ou de mauvais choix lexicaux dans les domaines médical, juridique, diplomatique, technique, médiatique.

\subsection{Déni de la traduction comme profession}

Le déni a porté également sur la traduction comme profession, notamment par les traducteurs eux-mêmes, qui ont intégré, incorporé, intériorisé les aspects «subalternes» de leur travail, pris entre l'idéalisme sacrificiel et le matérialisme calculateur de leur activité, assumant longtemps leur labeur, leur servilité, leur "vocation" toujours précaire, comme si ce métier ou cette pratique exigeait une prédisposition à l'effacement, à la docilité (Kalinowski, 2002; Simeoni, 1998), sinon à l'autodestruction (certains traducteurs se complaisant à se dénigrer entre eux ou à déprécier les solutions de leurs collègues).

Les métaphores de la traduction et les figures du traducteur dans l'imaginaire collectif sont aujourd'hui assez régulièrement reproduites dans les fictions romanesques, les films ou même dans les médias (Gambier, 2012). Elles frisent les stéréotypes, les clichés, le traducteur étant plus souvent vu comme un ermite besogneux, un marginal, un imposteur, un serviteur qu'un médiateur, un expert, un créateur. Les discours fatalistes et les représentations dévalorisantes ne datent pas d'aujourd'hui: dès la fin du Moyen Âge $\left(X V^{\mathrm{e}}\right.$ siècle), on trouve ces rapprochements avec le reflet, la pâle étoile, le revers de tapisserie, le caméléon, etc.

\subsection{Déni de la traduction comme discipline}

Le déni a enfin porté longtemps sur la traductologie comme discipline autonome, matière à réflexion. Encore aujourd'hui, son statut demeure ambigu dans les institutions universitaires, entre langues et littérature. D'ailleurs, bien des programmes de formation des traducteurs qui se sont multipliés ces dernières 
années hésitent à lui accorder une place, réduisant le métier à un ensemble de savoir-faire et de ficelles, inapte à l'auto-réflexivité. Une telle ambiguïté reflète le malaise des universités pour affronter l'interdisciplinarité, les communications interculturelles, la diversité linguistique, alors même que la mondialisation des affaires, les migrations lancent des défis à la plupart de nos sociétés.

Les ruptures entre le réel et les commentaires qu'on en fait, le double langage, le déni des réalités, parfois revers de l'idéalisme, souvent défense de ses intérêts, ne sont pas propres bien sûr à la traduction, aux traducteurs, à la traductologie. Les actualités récentes ont accumulé des exemples de déni comme comportement assez fréquent: déni du lien entre la pollution (au mercure, à l'amiante, à l'atome, au pétrole) et certaines maladies ou certains handicaps; déni des effets des contraintes financières et de la médiatisation sur les valeurs sportives ou olympiques; déni de la nécessité d'une politique économique commune tandis qu'on a créé une monnaie européenne commune, etc.

Les dénis mentionnés sur les besoins en traduction, sur le travail et sur la profession du traducteur ont la vie dure. Résisteront-ils aux transformations en cours (multiplication des appellations, diversification des pratiques, offres d'outils d'aide à la traduction) qui font du traducteur un individu de plus en plus "dématérialisé», non réductible à ses crayons et dictionnaires?

\section{Un nouvel environnement de travail}

En moins de deux décennies, on peut dire que l'informatique a fait bouger les lignes dans le monde de la traduction - transformant les ressources du traducteur et accélérant les possibilités de traduire. Des dénis de traduction, on passe à un désir de traduction, parfois même frénétique, par exemple chez les fansubs ou fandubs, qui s'approprient un film pour le sous-titrer ou le doubler dans les plus brefs délais.

\subsection{Virtualisation des outils partagés}

Les composants informatisés de cet environnement sont désormais nombreux. On peut citer les logiciels de mémoire de traduction, d'alignement de textes, de gestion de la terminologie; les correcteurs d'orthographe, de syntaxe, de ponctuation; les corpus électroniques et les possibilités de recherche qu'ils offrent; les outils de traduction automatique (TA); sans oublier 
la combinaison de différentes technologies, comme l'intégration de mémoires de traduction, de bases de données terminologiques et de TA, qui a permis le développement d'offres de traduction gratuites sur le Web. Sans oublier non plus le partage d'expériences grâce aux listes de diffusion, aux blogues ou encore aux médias sociaux, comme LinkedIn.

De l'utilisation des micro-ordinateurs qui a multiplié le partage des données et la mise en place de réseaux locaux, on passe maintenant à l'informatique dématérialisée (cloud computing), qui enlève au traducteur les soucis de gestion, de maintenance, de reconfiguration de ses moyens de travail: désormais, infrastructures, plateformes, logiciels, services et solutions sont accessibles, à distance, via le Net, et facturés selon l'utilisation (SaaS, software as a service ou logiciels services). Ce nouveau modèle de distribution en ligne d'outils partagés qui ne sont plus la propriété d'un seul utilisateur et ne sont plus installés (statiques) sur un ordinateur individuel pousse le traducteur à devenir membre d'une communauté internationale virtuelle et collaborative, puisque les mises à jour, les nouvelles versions sont immédiatement disponibles et que tous en bénéficient. De tels logiciels et services en traduction s'adressent à des traducteurs professionnels, amateurs, occasionnels ou encore à des agences, des institutions, des entreprises. Ils peuvent proposer quelques fonctionnalités comme la gestion de projet, la révision, la gestion de la terminologie, voire un environnement de travail complet. Parmi eux, gratuits ou payants, on peut penser à Translation Workspace, Wordbee, XTM Cloud, Google Translator Toolkit, Lingotek.

Cette évolution rapide n'est pas sans conséquences sur la pratique, sur l'organisation du travail et sans doute sur l'offre de traduction: dorénavant, les ressources accessibles en temps réel et mutualisées sont dynamiques, les coûts sont réduits (tout n'est plus à acheter, les tarifs éventuels peuvent être calculés à la demande ou selon l'utilisation à l'heure, à l'année, au volume de mots), la gestion est allégée (en termes de temps et de transparence), le travail est partagé. La dématérialisation favorise la simplification et la productivité de ce travail. En revanche, elle entraîne une certaine dépendance aux connexions à Internet et pose des problèmes de sécurité et de confidentialité. C'est le paradoxe de cette évolution: elle rejoint les défis des ordinateurs centraux des années 19501960, dévoreurs d'énergie et toujours risquant la panne. 


\subsection{Des traductions par les utilisateurs}

De ce qui précède, on ne peut conclure que les changements en cours se réduisent au seul développement de la traduction automatique, offerte gratuitement sur la Toile. On distinguera:

1) La traduction automatique via des logiciels disponibles sur le Net, où l'intervention humaine est limitée, sinon inexistante: on soumet un document à la TA pour en connaître l'essentiel, sans souci de qualité.

2) La traduction amateur, ${ }^{3}$ aussi automatisée, mais où l'utilisateur donne sa rétroaction, tente parfois d'améliorer les performances du logiciel de TA, sans avoir une formation particulière en traduction, à partir de ses intuitions linguistiques. On peut ici différencier deux types:

2a) La traduction par les fans (fantrad, scantrad, fansub, fandub), qui choisissent délibérément un manga, un film d'animation, un jeu vidéo... et le traduisent (le sous-titrent, le doublent) pour le faire connaître au plus vite à d'autres. Ces fans ne sont pas des professionnels de la traduction - d'où leur transgression de certaines conventions et normes respectées par les professionnels (par exemple, touchant les sous-titres, le nombre de lignes, la vitesse de défilement, la position, les caractères typographiques utilisés, l'ajout de gloses). Ils ne sont pas tous non plus des pirates, d'aucuns respectant les droits d'auteur et cessant de diffuser leur version traduite sur Internet dès que le livre ou le film sort officiellement.

2b) La traduction participative ou collective (crowdsourcing), ${ }^{4}$ employée par exemple pour localiser des logiciels ou des sites web, ou pour traduire des articles, des exposés, des textes littéraires, des interviews. Dans cet effort collectif non rétribué,

3. La terminologie est redondante et vague en anglais: community translation, collaborative translation, citizen translation, paraprofessional translation, user-generated translation, volunteer translation, sans oublier le CT3 (community, crowdsourced and collaborative translation) proposé par Common Sense Advisory, sont autant de termes employés pour désigner cette forme de traduction.

4. Crowdsourcing a été rendu de différentes manières en français, parmi lesquelles collecte d'informations par la foule, approvisionnement par la foule et collaboration de masse. 
les participants volontaires et anonymes (ou pas) recourent à leurs compétences linguistiques et à leur temps libre pour traduire une phrase, un paragraphe, une page... qui peuvent être retraduits, révisés par un autre, jusqu'à la finalisation de l'ensemble. Ces volontaires peuvent ne traduire qu'une fois ou des centaines de fois, grâce à des outils comme Traduwiki, Wikitranslate, Google Translate. Les médias sociaux ou réseaux socionumériques (Facebook, Twitter, LinkedIn, etc.) profitent de ce relatif engouement pour se rendre accessibles au plus grand nombre. Déjà, la traduction collective a donné lieu à des prises de position fortes, sous prétexte de la piètre qualité qu'elle offrirait et de la concurrence déloyale qu'elle ferait aux professionnels, puisqu'elle peut être utilisée de fait par des secteurs à but lucratif ou non. Quoi qu'il en soit, elle souligne que les limites entre outils/technologies créés pour les professionnels et ceux destinés au grand public s'estompent - ces derniers étant parfois adoptés et adaptés par les professionnels.

Deux remarques s'imposent ici: le volume des traductions possibles dépasse les capacités de tous les professionnels réunis; la traduction n'a pas le prestige de la musique, de la photographie, du journalisme ou du cinéma, qui attirent sur le Web des millions d'amateurs prêts à promouvoir sans compensation le produit de leur passion, de leur passe-temps. Longtemps déniée, la traduction n'appelle pas le même enthousiasme. On peut dire toutefois que les moyens d'aujourd'hui rendent la traduction désirable et faisable, mais que ce désir n'est pas fou. Ces moyens ne soulèvent pas les masses même s'ils permettent d'envisager de briser certaines barrières linguistiques, vu la quantité potentielle de documents à traduire. L'impact du crowdsourcing sur l'industrie de la traduction sera sans doute limité, en dépit de certains discours euphoriques actuels portant sur des cas très visibles.

3) La traduction collaborative (team work) effectuée par des professionnels sur un même document, mettant en commun des ressources de l'informatique dématérialisée, incluant la recherche documentaire et terminologique, et la relecture et la révision. Elle comprend des sites comme Proz et Translator's café. Le cloud ne saurait être confondu avec le crowd. 
4) La traduction avec logiciels libres (open source): ces logiciels ne sont pas nécessairement gratuits, mais peuvent être adaptés à certains besoins et être redistribués à d'autres. Ce type de traduction est le fait de professionnels, à plein temps.

5) La traduction bénévole par réseau, réalisée par des professionnels (c'est-à-dire des personnes qui ont une formation en traduction et/ou une expérience en traduction), et qui s'effectue par exemple via des réseaux comme Babels, ECOS, Traducteurs sans frontières, etc. (Gambier, 2007). Ces professionnels militants servent une cause explicite, répondent à des besoins exprimés par des $\mathrm{ONG}$, des associations. Leur réseau est associé à une activité sociale particulière ou à un engagement au nom de certaines valeurs.

Il y a donc une différence entre les types 1-2 et 3-5, où les professionnels partagent des outils, des problèmes, des solutions et mettent fin à un certain individualisme ou à une vision romantique $\mathrm{du}$ traducteur, reconfigurant leur corporation socioprofessionnelle grâce à l'offre technologique pour faire face aux défis de la délocalisation, de la compétition, de la précarité, des enchères en ligne, des appels d'offres internationaux. Pour 1-2, leur seul lien est technologique dans la plupart des cas, et leur intérêt commun porte sur un site, un réseau, un produit. Ces «communautés» en ligne sont donc plutôt à court terme et limitées dans leur teneur et leur portée. Ce qui rassemble les deux ensembles, c'est le décentrement vers l'acteur (traducteur, utilisateur), producteur de contenu. L'intelligence collective mise ainsi au service de la traduction a diverses motivations. Certains internautes sont des professionnels soucieux de développer leur métier, d'autres sont des activistes ayant une orientation idéologique marquée, d'autres, des amateurs technophiles, d'autres encore, des freelancers qui s'efforcent de trouver de nouvelles niches... L'évolution est donc à la fois technique, économique et sociale: elle est tantôt contrainte par la délocalisation, tantôt poussée par une production multilingue qui doit être plus rapidement accessible, tantôt stimulée par une certaine cause.

\section{Différenciation entre traducteurs ou entre traductions?}

Le crowdsourcing (une tâche de traduction est offerte à un groupe indéfini de volontaires) a suscité des inquiétudes quant aux personnes concernées (Sont-elles des traducteurs? Comment 
sont-elles récompensées pour leur travail?), quant à l'éthique (Quelles sont, par exemple, les implications de ce travail gratuit pour les entreprises qui recherchent avant tout le profit?), quant à la notion même de traduction (telle qu'elle est réalisée et/ou perçue).

\subsection{Amateurs, traducteurs « naturels »?}

Il faut souligner ici les problèmes méthodologiques pour mieux appréhender ces traducteurs volontaires - leurs motivations, leurs attentes, leurs représentations de la traduction, leurs langues de travail, leur profil socioéducatif, etc. Comment les reconnaître puis les joindre? Comment circonscrire une étude de cas qui se prolonge? Est-il possible d'enquêter sur l'identité et l'habitus de ces traducteurs en ligne?

On peut signaler ici la mésaventure survenue aux sociologues de l'Université Harvard, qui ont téléchargé des milliers de profils Facebook, puis diffusé leurs données, jusqu'à ce que certains se reconnaissent et portent plainte pour atteinte à la vie privée (Parry, 2011). Penser facilement rassembler une masse de données en ligne sans interagir avec quiconque, c'est d'abord ne pas oublier l'accord des intéressés pour analyser leurs blogues, leurs chats, leurs twittes - obtenir un anonymat absolu dans les réseaux ouverts étant quasiment impossible. Il y a là des défis éthiques et légaux qui s'ajoutent aux problèmes de collecte et d'analyse.

La sociologie dite des usages se focalise sur les pratiques des TIC, surtout à partir des années 1980 avec les premiers réseaux télématiques (voir par exemple la revue Réseaux). Comment et combien les aspects sociaux et les objets techniques se coconstruisent-ils? Â ce jour, on ne peut pas dire, cependant, que cette sociologie a beaucoup dépassé l'étude des traces, comme si la quantification de telles pratiques suffisait à comprendre ceux qui ont une position sociale, un métier, des sociabilités, lorsqu'ils s'adonnent au Net avec plusieurs pratiques. Par exemple, on ne consulte pas seulement Wikipédia; on a aussi d'autres pratiques documentaires et informationnelles. Bref, on a beaucoup de données sur ce qu'on fait sur Wikipédia, mais peu sur les autres usages, sur l'identité sociale des internautes; on a beaucoup de données sur la fréquence des courriels, le pourcentage de personnes qui commandent en ligne, qui utilisent des services de banque en ligne - avec statistiques, typologies, cartographies -, mais on 
demeure assez ignorant sur les dynamiques sociales et les sens pratiques dont sont porteurs les usagers.

Lobservation des pratiques, le recueil de déclarations, l'enregistrement des traces doivent encore être corrélés aux statuts et éléments identitaires des usagers, aux contenus produits en ligne. Il y a une certaine mystification derrière des mots comme "communauté», comme si tous ses membres étaient également compétents, tacticiens, explorateurs, avec les mêmes aptitudes interprétatives. L'idéologie de l'empowerment laisse croire que tous les amateurs sont, au même titre, autonomes, réactifs, maîtres de leurs mouvements, réflexifs.

Nombre de discours actuels sur la traduction collective participent de cet engouement technophile supposé, rabattant la communication, les usages sociaux de l'Internet sur une certaine mythologie de la société de l'information et du Web participatif. Ici la traductologie pourrait rejoindre la sociologie, en cherchant de nouveaux dispositifs d'enquête, en remettant en question les présupposés de déclarations sur la participation égale de tous, l'effacement des autorités, la démocratisation des communications, et en constatant les associations temporaires, intéressées, fragmentées sur le Web.

La distinction récurrente à propos des traductions collectives porte souvent sur les compétences des participants. Sont-ils des traducteurs amateurs, non professionnels, comme on les désigne parfois? De fait, les publications en traductologie s'attardent depuis quelque temps, surtout en étudiant les processus de traduction, sur des dichotomies floues: novices/professionnels, non professionnels/volontaires, traducteurs naturels/traducteurs formés, amateurs/experts, etc. Par ailleurs, le professionnalisme et la professionnalisation ont fait l'objet de réflexions récentes non seulement pour le traducteur, mais aussi pour l'interprète de communauté (Wadensjö et al., 2007; Sela-Sheffy et Shelsinger, 2009; Sela-Sheffy et Shelsinger, 2010; Katan, 2011; Wadensjö, 2011). Les critères d'identification des deux notions sont multiples: l'une fait appel à des compétences, des savoirs, à une expérience, une pratique régulière, une efficacité, une précision, à une éthique... tandis que l'autre englobe des efforts individuels et collectifs pour atteindre un certain statut, définir des normes de bonnes pratiques, contrôler l'accès à la profession, à la formation et aux offres de travail, etc. La masse éventuelle de données à 
rassembler et à traiter ne fait-elle pas s'éloigner de plus en plus un système d'accréditation des traducteurs?

Quoi qu'il en soit, le volontaire qui traduit sur le Web peut être un fan, un expert, un activiste, tantôt avec une expérience et/ ou une formation en traduction, tantôt sans elles. ${ }^{5} \mathrm{Il}$ peut coopérer avec un professionnel. Cependant les fournisseurs de technologie ne relèvent pas de la même "communauté» que ces utilisateurs: Google, Facebook... font du profit, sont cotés en bourse avant d'être «médias sociaux».

Il resterait à voir comment et jusqu'où ces nouvelles pratiques déqualifieraient, sinon déprofessionnaliseraient les traducteurs à temps plein, formés et riches d'une certaine expérience, et également comment et jusqu'où elles pourraient aider au développement des compétences en traduction. Les possibilités technologiques peuvent susciter de nouveaux problèmes, mais aussi offrir de nouvelles possibilités et niches, inexistantes auparavant.

\subsection{Vers un futur bigarré ?}

Avec les développements décrits ci-dessus, productivité, accessibilité, qualité, flux collaboratif sont plus étroitement imbriqués, et plutôt que de focaliser le débat sur la tension, sinon l'opposition présumée entre professionnels et amateurs, il serait plus urgent et opportun d'organiser le dialogue entre les traducteurs et les fournisseurs d'outils. En effet, avec quelquesuns de ces outils, on constate une certaine régression, un retour à une conception ancienne de la traduction basée sur le mot, le mot à mot - comme si elle (re)devenait simple transfert formel, mécanique, comptable -, conception qui l'a fait être niée pendant si longtemps ( $c f .1)$. Les traductions juxtalinéaires des directives de l'Union européenne, celles réalisées avec les contraintes des mémoires de traduction, le sous-titrage en direct, les sous-titres de fans... tendent à coller à la source, à se faire verbatim, sans s'inquiéter outre mesure des effets sur leur réception, leur lecture. Avec le changement des conditions et du rythme de travail, cette tendance peut démotiver le traducteur, le déposséder de tout

5. Les graffeurs, par exemple, représentent également une diversité souvent niée, sous prétexte que tous les graffitis seraient illégaux, quel que soit leur auteur: aux jeunes de milieu aisé ou non se joignent des étudiants de BeauxArts, des autodidactes, des dessinateurs professionnels... Les artistes de la rue comme les traducteurs ne sont pas un groupe homogène. 
pouvoir, forcé d'être toujours en ligne et soumis à l'outil imposé par le client. Le désir de traduction, quasi compulsif chez les fans, par exemple, et plutôt issu d'un partage raisonné chez les bénévoles, serait pluriel, avec des degrés mesurés et reflétés par les modalités de travail. N'empêche, ne modifie-t-il pas en profondeur les figures du traducteur, même si parfois et paradoxalement désir et déni se rejoignent?

Lindividualisme traditionnel des traducteurs ne doit pas occulter cependant le travail par paires ou en groupe, depuis le $X V I^{e}$ siècle à aujourd'hui, avec par exemple la nouvelle traduction de la Bible (2001) coordonnée par Frédéric Boyer, par tandem exégète + écrivain, ou encore la nouvelle traduction d'Ulysse de James Joyce en 2007 par une équipe de sept traducteurs qui se sont rencontrés régulièrement, sans oublier les projets de localisation impliquant une division du travail à plusieurs, en contact réel ou virtuel.

La société numérique ne se définit pas comme un collectif structuré par des organismes médiateurs (partis, syndicats, associations): elle est plutôt un ensemble de micro-unités. Dans ce contexte, la traduction ( $c f .2 .2)$, comme d'autres produits et services, prend une nouvelle dimension grâce aux logiciels libres, au modèle de distribution en ligne d'outils partagés, faisant place au partage, à la collaboration, à l'accessibilité, au volontariat, et annonçant un futur plus bigarré entre professionnels, hyper-spécialistes et amateurs travaillant au coup par coup, sans nécessairement de continuité, en réseau transitoire. Les usagers, consommateurs de traduction, peuvent désormais en être aussi les producteurs (Perrino, 2009). Il est évident que le statut et les représentations du traducteur s'en trouveront changés.

À défaut d'enquête sur l'identité et les profils de ces traducteurs en ligne, encore difficile à mener ( $c f .3 .1)$, on peut s'attarder sur le devenir des journalistes, confrontés aussi à l'informatisation et à l'amateurisation. Le parallèle ne permet pas néanmoins d'extrapoler hâtivement. Journalistes et traducteurs ont des points communs (Gambier, 1994, p. 76-77) : ils travaillent sur des formes écrites et orales et ont une responsabilité socioculturelle qui dépasse la production immédiate d'énoncés. Ils ont besoin d'aptitude à la recherche documentaire et terminologique; ils doivent savoir établir des relations avec d'autres experts. L'efficacité communicationnelle des uns peut servir aux autres tandis que le souci de la qualité, de la 
précision des traducteurs pourrait aider les gens des médias appelés de plus en plus à traduire à vue, à synthétiser des textes. Dans les deux cas, l'apprentissage d'une démarche est plus important que l'acquisition de savoirs vite obsolètes, et l'autonomie de décision ainsi que la capacité à s'autoévaluer apparaissent fondamentales. Enfin, les deux professions sont confrontées aux apports des TIC et aux transformations qu'elles impliquent dans la production et la distribution des informations.

Des premières révélations de WikiLeaks (juillet 2010) à la fermeture de News of the World (juillet 2011), on a un résumé des changements rapides du journalisme. Au modèle pyramidal de la salle de rédaction (du directeur qui décidait de la ligne du journal aux rédacteurs en chef, assistés de reporters et de journalistes spécialisés et de pigistes, l'ensemble étant appuyé par les correcteurs, les linotypistes et les ouvriers des rotatives) succède un "plateau» numérique où circule un flux d'informations envoyées et mises à jour de manière continue, où moins de journalistes travaillent pour plus de "tuyaux» (magazine hebdomadaire, journal quotidien gratuit ou payant, sites Internet, application sur téléphone mobile) avec des données textuelles, visuelles et sonores. La rédaction ne vit plus au rythme du bouclage, mais plutôt des modes de consommation de l'information par les lecteurs. Ces derniers peuvent participer, comme les journalistes qui transforment et formatent les dépêches des agences de presse et des reporters, à la production de l'information, en envoyant images, vidéos, commentaires. Désormais la presse est en concurrence avec les services agrégateurs d'info en ligne, gratuits ou à bas prix. Cette évolution rapide véhicule un certain nombre de mythes, par exemple sur la puissance des médias sociaux (comme s'ils étaient tous fiables au même degré), sur l'agonie $\mathrm{du}$ journalisme (sous prétexte de démocratisation apparente de l'accès aux informations). Les illusions techno-utopiques n'empêchent pas la baisse de diffusion des journaux, la diminution des revenus publicitaires, les licenciements dans les rédactions, ${ }^{6}$ la collusion entre médias, pouvoir et argent, le remplacement $\mathrm{du}$ journalisme d'investigation par des pages d'opinion, le défi des droits intellectuels, moraux et financiers des journalistes,

6. Près d'un tiers des journalistes professionnels ont été licenciés aux ÉtatsUnis ces dix dernières années, et 3000 postes ont été supprimés en France, en 2010. 
etc. L'accumulation des tâches, la tyrannie de la réactivité, la redondance des contenus, la multiplication des supports pour un article, l'impact du téléphone et de l'ordinateur portable, ainsi que l'émergence de nouvelles sources d'information créent à la fois une profession en alerte constante et un journalisme sans journalistes les deux étant soumis aux diktats de l'immédiateté, de la vitesse et du marché. Dans ces conditions, on peut s'interroger sur le devenir de la presse de qualité et sur la crédibilité des informations diffusées, sur la confiance à leur accorder.

Les avatars du journalisme ne croisent-ils pas ceux de la traduction? Les internautes qui contredisent, complètent, discutent une information ne ressemblent-ils pas à ceux qui traduisent sur le Net pour faire connaître un document, un film? Les contradictions entre une minorité de journalistes acoquinés avec des politiciens et la majorité des précaires ne ressemblentelles pas à celles qui existent entre quelques traducteurs littéraires «renommés» et la masse des petites mains qui traduisent au quotidien? Les peurs suscitées par les TIC dans la transformation des conditions de travail ne sont-elles pas similaires chez les journalistes et les traducteurs? Les deux métiers, changeant sous la pression technologique et financière, ne sont-ils pas obligés de se réinterroger sur leurs normes, leur éthique? Les amateurs, longtemps décriés dans les milieux professionnels, ne tiennent-ils pas leur revanche? De marginalisés, caricaturés (figure du pirate radio, du fan aliéné, de l'adolescent hacker irresponsable), ces amateurs contraignent à redéfinir contours et missions de certaines professions. Qu'on s'en plaigne comme une forme (déguisée?) du libéralisme ou qu'on les loue parce qu'ils vivifient certaines pratiques, ne reflètent-ils pas les profondes mutations induites par les TIC?

\section{Implications de la pluralité des acteurs et des pratiques}

Avec les développements mentionnés jusqu'ici, deux pistes de réflexion souvrent: celle de la dimension économique de la traduction et celle des compétences mises en œuvre. Dans le premier cas, il ne s'agit pas de renforcer l'hégémonie supposée du modèle économique dans les sciences sociales, mais davantage de comprendre certaines transformations des pratiques traductionnelles dans une perspective toujours négligée en traductologie: la traduction, service rétribué ou gratuit, ne se situe 
pas en dehors de la sphère marchande, même si elle a d'autres dimensions (idéologique, culturelle, identitaire). Dans le second cas, il s'agit de savoir à quelles conditions d'expertise minimale la traduction est faisable. Entre le professionnel hyperspécialisé, pris dans un réseau international, et un amateur très occasionnel, n'y $\mathrm{a}-\mathrm{t}$-il aucune convergence?

\subsection{Vers un tournant économique en traductologie}

La traductologie a connu bien des tournants en trois décennies (linguistique, culturel, idéologique, sémiotique, cognitif, sociologique, etc.), tournants qui donnent un peu le tournis, comme si cette boulimie de virages, de tours et détours, cette vitesse pour changer d'orientations étaient plutôt une conduite en état d'ivresse. Dans cet ensemble manque toujours le chaînon économique, déjà déploré par Pym et al. (2006, p. 2), c'est-àdire la question des coûts, des investissements, des modes de paiement. De l'agence multinationale souvent gérée par un non-traducteur à la maison d'édition soucieuse de conquérir de nouveaux marchés (Heilbron et Sapiro, 2002; Sapiro, 2008 et 2009), de l'institution internationale ou gouvernementale rétribuant un service de traduction à l'ancien enseignant qui fait payer au noir ses traductions pour arrondir ses fins de mois, les dimensions économiques et financières ne sauraient davantage être négligées, comme facteur orientant, sinon déterminant, certains choix et décisions. Il ne s'agit pas d'imposer un modèle économique aux échanges traductionnels, de les réduire à des marchandises, mais de comprendre les effets des conditions de travail, les transformations des pratiques. On a déjà signalé ( $c f$. 1.1) les lacunes portant sur notre (mé)connaissance du marché, pluriel, évolutif, distinct, selon les volumes de la demande, les moyens utilisés pour satisfaire cette demande, et la nature de la relation qui lie le traducteur à son commanditaire (Gouadec, 2002 et 2007).

Autre aspect économique non négligeable: comment les compétences en langues étrangères affectent-elles les performances des entreprises ou, en d'autres termes, comment une politique linguistique, souvent implicite, a-t-elle un impact sur les politiques de traduction, souvent non dites? Une enquête internationale menée en 2008 sous la direction du Centre national des langues britannique (National Centre for Languages) et 
commanditée par la Direction générale de l'Éducation et de la Culture de la Commission européenne révèle que $11 \%$ des PME européennes exportatrices (945 000 firmes) perdent des affaires à cause des barrières linguistiques, ignorant que le russe, l'allemand et le polonais sont utilisés dans l'Europe orientale, que le français l'est dans nombre de pays africains et que l'espagnol est courant en Amérique latine! Moins de la moitié de ces entreprises ont envisagé une approche stratégique de communication multilingue (recrutement de natifs, adaptation de leur site web, recours à des agents locaux, offre de cours de langue au personnel, emploi de traducteurs/interprètes).

D'autres analyses, sur d'autres marchés, seraient bienvenues, par exemple sur les politiques linguistiques et les marchés des jeux vidéo, les divers types d'agences de traduction, non seulement selon leur taille et leur chiffre d'affaires, mais aussi selon leur manière de promouvoir leur marque, leurs services, leurs spécialisations, et comment leur marketing a évolué au cours des dix à quinze dernières années. Mais à ces études de macro-niveau devraient s'en ajouter de micro-niveau ( $c f$. Mossop, 2006), et ce, quel que soit le domaine sur lequel portent les traductions (médical, technique, commercial, juridique, audiovisuel, littéraire) :

- sur la comparaison des coûts entre la traduction/interprétation et les autres moyens de gérer les communications multilingues internationales ( $c f$. introduction);

- sur l'empreinte écologique d'un traducteur à l'Ouest et d'un traducteur en Inde, tous deux traduisant un même texte, ou sur celle d'un interprète qui voyage et d'un interprète qui fonctionne en vidéoconférence (coût, productivité et environnement);

- sur la traduction comme business proprement dit, notamment sur ses coûts en rapport avec les délais et les exigences de qualité ;

- sur les dépenses de fonctionnement et de dysfonctionnement de l'organisation de la traduction dans une entreprise commerciale, bancaire, etc.;

- sur les effets financiers des systèmes de mémoire de traduction quand ils accroissent la productivité ou au contraire l'entravent, faute de correspondants ad hoc entre les segments, quand il faut modifier, corriger ces systèmes ou que ceux-ci sont partagés 
à plusieurs, ou quand il faut réviser de façon approfondie la traduction;

- sur les coûts et les implications financières de l'utilisation de logiciels de traduction assistée ou de traduction automatique, avec ou sans pré-/post-édition;

- sur les comparaisons des modes de paiement des traducteurs (au mot, à la ligne, à la page, à l'heure, au nombre de lecteurs (du livre traduit) ou de visiteurs de sites web;

- sur les retombées économiques des changements dans la division du travail, avec de nouvelles tâches, nouvelles procédures, nouvelles manières dans les prises de décision, nouveaux rapports au document de départ (achevé ou en cours de rédaction);

- sur les conséquences financières des localisations, réussies ou pas, de sites web;

- sur les coûts de la révision et de la relecture, selon leur place et leur fréquence dans le processus de travail et les attentes suscitées (par exemple, révision en interne alors que la traduction est externalisée);

- sur l'impact financier du recours au seul anglais dans les communications internationales d'une entreprise (par exemple, l'effet d'une publicité ou d'un slogan sur les ventes);

- sur les modes de sélection et de recrutement des traducteurs indépendants dans une agence de traduction, dans une entreprise... et sur les modes d'évaluation des services qu'ils rendent;

- sur les coûts et les effets de l'emploi d'un interprète de communauté, qualifié ou amateur, dans les consultations médicales ( $c f$. Ribera et al., 2008);

- sur les rapports entre les contraintes financières et d'une part les retraductions, d'autre part les adaptations, avec coupes et ajouts, de pièces de théâtre, de $\mathrm{BD}$, de livres pour enfants, de publicités.

Depuis les marchés de l'offre et de la demande jusqu'aux effets de la technologisation, depuis l'organisation du travail au quotidien jusqu'aux conséquences des fusions d'entreprises (des cultures d'entreprise), la palette est large pour traiter des dimensions économiques et financières des traductions et interprétations, 
des métiers de la traduction. Il y a un défi d'interdisciplinarité entre traductologie et études commerciales qui est à peine relevé aujourd hui malgré son urgence, puisque nombre de décideurs ne comprennent que le langage de l'argent. Un tel défi commande de remettre en question les formations des traductologues qui, on le voit bien, sont pris entre les retombées des technologies sur le travail du traducteur et les effets des communications multilingues à l'ère de la mondialisation.

\subsection{Expertise}

Les différentes formes et possibilités de traduction - de la quasi automatisée à la participative, en passant par la spécialisée et professionnelle - obligent à se demander si l'on a toujours affaire au même concept de traduction. Les moyens électroniques exigent sans cesse plus de liens entre les agents du processus, avec parfois pré- et/ou post-édition, révision, relecture, coordination des choix terminologiques, souci de la cohérence finale du produit. Mais y $\mathrm{a}-\mathrm{t}-\mathrm{il}$, dans tous les cas, une dynamique communicationnelle qui justifierait les décisions, les interventions du traducteur? Cette dynamique, avec objectif, possibilités de négociation, est-elle toujours explicite? Traduire sur le Net une conférence, en ignorant les conditions dans lesquelles elle a été tenue et l'auditoire alors visé, est-ce comme traduire un manuel sur le harcèlement scolaire quand on connaît les raisons pour lesquelles l'ouvrage a été rédigé et qu'il est à traduire?

Peut-on gérer la coopération, la confiance entre anonymes, dans un système basé sur l'efficacité, la flexibilité, l'asymétrie des rapports entre les personnes engagées, et donc d'autorité et de responsabilité asymétriques, comme on gère le travail d'une équipe dans laquelle on connaît les compétences de chaque membre sélectionné? Faire appel aux anonymes pour un travail gratuit peut-il se comparer à un recrutement aux critères explicites pour un travail rémunéré? Une approche trop centrée sur la production, l'industrie de la traduction ou encore une approche trop économiste ( $c f .4 .1)$ pourraient oblitérer de telles questions.

Souvent dans une perspective de formation, on a étudié en traductologie, ces deux dernières décennies, le passage du novice au professionnel, le développement des compétences, le type de connaissances et de comportements (notamment dans la prise de décisions) dans ces deux groupes, pas toujours faciles à différencier. 
Peut-on recourir aux mêmes méthodes pour appréhender la diversité actuelle des traducteurs? Devrait-on d'abord se focaliser sur le processus ou sur les profils, les habitus, les perceptions, les autoreprésentations de ces différents types de traducteurs actifs pour répondre aux questions posées ci-dessus? Pour reprendre le parallèle avec les médias, on peut affirmer qu'on se préoccupe souvent de leur indépendance, mais beaucoup moins de celle des experts (économistes, politologues, etc.) qui interviennent, mais pas uniquement, dans ces mêmes médias. En est-il de même en traductologie? Faut-il se contenter d'observer, de décrire les actes des seuls traducteurs, et négliger les actes de ceux qui interviennent en amont (ingénieurs en langue, informaticiens en TA, fournisseurs de services de traduction) et en aval (ceux qui décident par exemple d'arrêter une traduction en cours, de la mettre en ligne, de la diffuser)?

Parmi les compétences diverses du traducteur, dont la liste n'est nulle part exhaustive (compétences linguistique, culturelle, technique, etc.), l'une semble importante, quel que soit le degré d'engagement et de professionnalisation de ce traducteur: celle de la lecture et de la compréhension de ce qu'il faut traduire, compétence qui fait appel à des connaissances antérieures, à la mémoire (à court et à long terme), aux capacités logiques d'inférence. Là encore, un certain nombre de questions se posent qui pourraient modifier nos comportements et donc différencier les traducteurs selon leur milieu socioculturel d'origine, leurs habitudes et capacités d'apprentissage. Le Net favorise une lecture fragmentée (par liens successifs) et rapide (recherche d'informations ponctuelles). De même, les traductions produites par exemple par Google Translate sont de qualité «suffisante» parce qu'on les consulte plus qu'on ne les lit, les assimile. D'aucuns pourraient parler de lecture et d'écriture «superficielles». Est-ce à dire que le Web laisserait au traducteur expert ses aptitudes les plus pointues: la lecture en profondeur, la rédaction des textes pertinents, et pas seulement la révision, l'édition de documents générés par l'ordinateur? Les outils électroniques ne supprimeraient donc pas le traducteur qualifié. Une nouvelle hiérarchie de traducteurs s'imposerait, mais en haut ne se trouverait peut-être plus le traducteur littéraire comme cela a été le cas des décennies durant. Ce qui finalement troublerait aujourd'hui le milieu des traducteurs, ce ne serait pas tant l'introduction de plus en plus forte de l'automatisation que la 
perte de prestige de la littérature, longtemps valeur de référence de nos cultures lettrées et véhicule de mythes comme celui du génie de l'écrivain, de la créativité.

\section{En guise de conclusion}

Plusieurs phénomènes semblent incontournables aujourd'hui:

- l'omniprésence des technologies dans la majorité des productions et des services;

- la demande accrue du travail des traducteurs et interprètes, même si celui-ci reste souvent invisible, non ou mal reconnu;

- la nécessité de sortir de la bulle de la corporation des traducteurs parce que la traduction - ce qu'elle demande, ce qu'elle implique, ses effets, ses défis - touche plus que les traducteurs. Un ouvrage comme How to Translate for Dummies/ Comment traduire pour les nuls ne serait pas une provocation, car de plus en plus d'individus sont concernés par les échanges de données, d'informations, de savoirs, et par la diversité de leurs sources possibles;

- le volume à traduire dépasse certainement les capacités de travail des professionnels qui ont reçu une formation idoine.

Les dénis de traduction, violents, récurrents, sont anciens. Peut-on pour autant affirmer qu'ils sont désormais dépassés par le désir de traduire? Longtemps refoulée, la traduction serait désirable grâce à tous les moyens qui en facilitent l'offre. S'agit-il d'une simple inversion stimulée par les TIC ou d'une modification profonde du rapport à la traduction, alors que la globalisation s'accélère et que la dictature de l'urgence s'impose dans la plupart de nos activités? Le manque de recul et de moyens pour décrire et évaluer aussi complètement que possible la situation n'autorise pas à répondre de manière tranchée, d'autant que les fantasmes de toute-puissance d'accessibilité totale et de réalisation automatique marquent encore l'étape actuelle de l'évolution.

Il n’empêche, comme Daniel Simeoni l'avait suggéré (1998), les processus d'acquisition de l'habitus des traducteurs (quel que soit leur statut sociosymbolique) restent une problématique centrale, d'autant plus sensible que se développent désormais des structures de sociabilité particulières, avec les réseaux provisoires, ciblés, motivés. Ces structures transforment les rapports hiérarchiques traditionnels entre commanditaires et traducteurs ainsi qu'entre 
traducteurs eux-mêmes. Elles transforment également la mise en œuvre des normes de traduction et leur transgression, et le rapport à son travail, aux documents à traduire. Elles exigent enfin une approche interdisciplinaire dont les méthodes et concepts restent à préciser, à affiner. Le programme de recherche établi par Daniel Simeoni (1998, p. 21-24) prend ici un nouvel élan, tant les pratiques se diversifient, les expériences se multiplient, quitte même à se frotter à l'«impensé géopolitique de la théorie» (Simeoni, 2008, p. 337), car ces pratiques et expériences s'universalisent.

\section{Références}

Berman, Antoine (1990). «La retraduction comme espace de la traduction ». Palimpsestes, 4, p. 1-7.

Delisle, Jean et Judith Woodsworth, dir. (2007). Les traducteurs dans l'histoire ( $2^{\mathrm{e}}$ éd. rev. et corr.). Ottawa, Les Presses de l'Université d'Ottawa. [1 ${ }^{\text {re }}$ éd.: 1995 ]

Gambier, Yves (1994). «Multimédia et médiation: quels défis?» In Y. Gambier et M.Snell-Hornby, dir., Problemi e tendenze della didattica dell'interpetazione e della traduzione, Actes du colloque de Misano Adriatico, 28-30.9.1994. Numéro spécial de Koiné, IV, p. 67-79.

Gambier, Yves (2007). «Réseaux de traducteurs/interprètes bénévoles». Meta, 52, 4, p. 658-672.

Gambier, Yves (2012). «Le traducteur défiguré?» In E. Skibínska et N. Paprocka, dir., Figure(s) du traducteur. Wroklow, PU (coll. «Romanica Wratislaviensia», vol. 59), p. 13-24.

Gouadec, Daniel (2002). Profession: Traducteur. Paris, La Maison du traducteur.

Gouadec, Daniel (2007). Translation as a Profession. Amsterdam/ Philadelphia, John Benjamins.

Heilbron Johan et Gisèle Sapiro, dir. (2002). Traduction: les échanges littéraires internationaux. Actes de la recherche en sciences sociales, 4, 144.

Kalinowski, Isabelle (2002). «La vocation au travail de traduction». Actes de la recherche en sciences sociales, 4, 144, p. 47-54.

Katan, David (2011). "Status of Translators». In Y. Gambier et L. Van Doorslaer, dir., Handbook of Translation Studies. Amsterdam/ Philadelphie, John Benjamins, p. 146-152.

Lambert, José (1989). «La traduction, les langues et la communication de masse. Les ambiguïtés du discours international». Target, 1, 2, p. 215-237. 
Mossop, Brian (2006). «Has Computerization Changed Translation?» Meta, 51, 4, p. 787-792.

Parry, Marc (2011). «Harvard Researchers Accused of Breaching Students' Privacy». The Chronicle of Higher Education, 10 juillet 2011. [http://chronicle.com/article/Harvards-PrivacyMeltdown/128166/]

Perrino, Saverio (2009). «User-generated Translation: The Future of Translation in a Web 2.0 Environment». JoSTrans. The Journal of Specialised Translation, 12. [http://www.jostrans.org/issue12/art_ perrino.php]

Pym Anthony, Miriam Shlesinger et Zusanna Jettmarová, dir. (2006).

Sociocultural Aspects of Translating and Interpreting. Amsterdam/ Philadelphie, John Benjamins.

Ray, Rebecca et Nataly Kelly (2010). Reaching New Markets through

Transcreation. When Translation Just Isn't Enough. Lowell [Massachusetts], Common Sense Advisory, Inc., 47 p.

Ribera, Joan Muela et al. (2008). Is the Use of Interpreters in Medical Consultations Justified? A Critical Review of the Literature. Pass International v.z.w, 29 p.

Sapiro, Gisèle, dir. (2008). Translatio. Le marché de la traduction en France à l'heure de la mondialisation. Paris, Éd. du CNRS.

Sapiro, Gisèle, dir. (2009). Les contradictions de la globalisation éditoriale. Paris, Nouveau Monde éditions.

Sela-Sheffy, Rakefet et Miriam Shlesinger, dir. (2009). Profession, Identity and Status: Questions of Status and the Field, Part 1. Numéro spécial de Translation and Interpreting Studies, 4, 2.

Sela-Sheffy, Rakefet et Miriam Shlesinger, dir. (2010). Profession, Identity and Status: Questions of Role and Identity, Part 2. Numéro spécial de Translation and Iterpreting Studies, 5, 1.

Simeoni, Daniel (1998). "The Pivotal Status of the Translator's Habitus». Target, 10, 1, p. 1-39.

Simeoni, Daniel (2008). «Norms and the State: The Geopolitics of Translation Theory». In A. Pym, M. Shlesinger et D. Simeoni, dir., Beyond Descriptive Translation Studies. Investigations in Homage to Gideon Toury, Amsterdam/Philadelphie, John Benjamins, p. 329342.

Toury, Gideon (1995). Descriptive Translation Studies and Beyond, Amsterdam/Philadelphie, John Benjamins.

Wadenjsö, Cecilia (2011). «Status of Interpreters». In Y. Gambier et L. van Doorlaers, dir., Handbook of Translation Studies, Amsterdam/ Philadelphie, John Benjamins, p. 140-145. 
Wadenjsö, Cecilia, Birgitta Englund Dimitrova et Anna-Leena Nilsson, dir. (2007). The Critical Link 4: Professionalisation of Interpreting in the Community. Amsterdam/Philadelphie, John Benjamins.

\section{Yves GAMBIER}

Université de Turku 20014-Turun Yliopisto

Fi-Turku

FINLANDE

yves.gambier@utu.fi 